initiation of PN. Simple remedial actionwas introduced and the audit cycle was completed to assess the impact of mandatory minimal set documentation prior to initiation of $\mathrm{PN}$.

Methods A mandatory request sticker was introduced which prompted the clinical team to document why enteral nutrition is inappropriate and duration of inadequate enteral intake, the clinical purpose and goal of $\mathrm{PN}$ and expected duration of $\mathrm{PN}$. A prospective re-audit was performed to assess any change over the same six month period.

Results The initial audit included 43 patients and 34 patients received $\mathrm{PN}$ during the reaudit period. Administration of $\mathrm{PN}$ was predominantly in surgical $(47 \%)$ or critical care $(20 \%)$ areas. The indication was documented in the case notes in $88 \%$ of cases (initially $43 \%$ ), and considered appropriate by the nutrition team in $94 \%$ of cases (initially $67 \%$ ). There were unreasonable delays in $21 \%$ of cases (initially $29 \%$ ), mostly related to delays in placing appropriate central venous access. The treatment goal was documented in $82 \%$ of cases (initially $36 \%$ ). Alternatives were considered in $100 \%$ of cases (initially $64 \%$ ) and an appropriate assessment was made in $97 \%$ of cases (initially $69 \%$ ). TPN was reviewed and monitored appropriately in the initial audit and there was no change in the re-audit. Metabolic complications occurred in 38\% of cases (initially 52\%), all cases were managed appropriately. Nine percent of patients received PN for fewer than 5 days (initially $12 \%$ ) but the vast majority received an appropriate course length. Seventy four percent of patients were weaned onto oral diet (initially 67\%) and 6\% of patients died during their stay (initially $17 \%$ ).

Conclusion A relatively simple intervention, involving no additional cost, vastly improved the delivery of PN in our hospital. Mandatory information prior to starting helped to focus the thoughts of the requesting clinicians to decide on the appropriateness of using PN. This simple win-win strategy can be easily adopted in other hospitals to improve delivery of PN.

Disclosure of Interest None Declared.

\section{PWE-176 TRENDS IN GASTROSTOMY INSERTION OVER A 10 YEAR PERIOD. ARE THE INDICATIONS CHANGING?}

doi:10.1136/gutjnl-2013-304907.464

1."C S MacLeod, 'R McKay, ${ }^{1} \mathrm{D}$ Barber, ${ }^{1} \mathrm{~A}$ W McKinlay, ${ }^{1} \mathrm{~J}$ S Leeds on behalf of Aberdeen PEG research group. 'Gastroenterology, Aberdeen Royal Infirmary, Aberdeen, UK

Introduction Gastrostomy insertion is a well accepted technique for enteral feeding however the most robust evidence is for patients with dysphagic stroke or head and neck cancer. Other indications for gastrostomy insertion have developed but the proportion of patients referred for each indication is not well documented. Our aim was to examine referral patterns for feeding tube insertion in our unit over the last ten years with reference to indication for tube insertion and type of tube inserted.

Methods A retrospective review of all gastrostomy insertion was performed over the period May 2001 to December 2011 using the gastrostomy database. Data collected included patient demographics, indication for tube insertion and type of tube inserted (PEG, PEJ, PEG-j or gastropexy). Indication was divided into 5 main categories; stroke with dysphagia, head and neck cancer, neurological, dementia and other. Assessment of total number of each type of tube inserted per year and, for PEG alone, the total number of insertions per indication per year.

Results During the study period 1387 tube insertions were carried out (1289 PEG, 41 PEJ, 32 PEG-j and 25 gastropexy). Over the ten year period there was a significant increase in the number of all types of tubes inserted $(p<0.001)$ with 65 PEG's alone inserted in 2001 rising to 129 PEG's, 7 PEJ, 4 PEG-j and 11 gastropexy's in 2011. In 2001 the proportion of patients having a PEG inserted by indication was stroke with dysphagia $26.2 \%$, head and neck cancer $6.2 \%$, neurological $35.4 \%$, dementia $1.5 \%$ and other $10.8 \%$ (indication was not recorded in 20\%). In 2011 the proportion of patients having a PEG inserted by indication was significantly different with stroke with dysphagia (22.5\%), head and neck cancer (12.4\%), neurological $(37.2 \%)$, dementia $(0.0 \%)$ and other $25.6 \%$ (indication was not recorded in $2.3 \%$ ). The main differences were an increase in insertions in those with head and neck cancer and other indications. Conclusion In our centre, there has been a significant increase in the number and type of feeding tubes inserted over a decade. The proportion of patients having a PEG for the traditional indications such as stroke account for around one third of all insertions. PEG insertions for the miscellaneous indications have significantly increased suggesting that longer term nutritional support is being offered for a much broader range of conditions.

Disclosure of Interest None Declared.

\section{PWE-177 AN AUDIT TO EVALUATE THE SAFETY AND EFFICACY OF PERCUTANEOUS ENDOSCOPIC GASTROSTOMY (PEG) PLACEMENT IN PATIENTS WITH LEARNING DISABILITIES}

doi:10.1136/gutjnl-2013-304907.465

1,"C Black, "I Shaw, 'L Ayres. 'Gastroenterology, Gloucestershire Royal Hospital, Gloucestershire Hospitals NHS Foundation Trust, Gloucester, UK

Introduction Guidelines exist to aid clinicians in selecting patients likely to benefit from percutaneous endoscopic gastrostomy (PEG) placement. Patients with learning disabilities (LD) are distinct from those with dementia in whom PEG placement in not usually recommended ${ }^{1}$. We report our experiences of PEG placement in patients with learning disabilities and our use of a specially designed pathway which emphasises multi-disciplinary team (MDT) input.

Methods To describe the efficacy and safety of PEG insertion in patients with $\mathrm{LD}$, we retrospectively analysed a cohort of 42 patients with severe LD who required nutritional support. We reviewed their medical case notes and their dietetic notes.

Results Forty two patients underwent 117 procedures: 38 index PEG insertions, 43 PEG replacements, 35 percutaneous endoscopic gastrojejunostomy (PEGJ) replacements and 1 PEG removal (6 procedures $(5.1 \%)$ were unsuccessful). Best interest meeting or MDT assessment took place in all patients. General anaesthetic was required for 34 (29\%) procedures. Mean weight pre-PEG was $39.8 \mathrm{Kg}$ and mean weight after a median follow-up of 21.5 months was $46.1 \mathrm{Kg}$ (mean overall weight gain $=2.3 \mathrm{~kg}$ ). 30 day mortality was zero. Complications were common $(74 \%)$ and included perforation $\mathrm{n}=1$, aspiration pneumonia $\mathrm{n}=13$, tube displacement $\mathrm{n}=10$, PEG site infection $\mathrm{n}=4$. In 2 cases of unsuccessful PEG insertion surgical feeding gastrostomy was required. On-going vomiting occurred in 6 patients $(14.3 \%)$ prompting conversion to PEG-J in 5 cases.

Conclusion We found that PEG placement in LD patients was a positive intervention for the majority. Although there were frequent late complications such as aspiration pneumonia and PEG displacement, these reflect the complex underlying medical and social needs of the patients concerned. Overall, PEG placement appears to be safe and results in a modest weight gain; PEG-J should be considered for those with on-going vomiting. The use of a pathway for PEG placement ensures MDT input, appropriate patient selection, better pre-procedure planning e.g. use of general anaesthesia, and appears to promote good patient outcomes.

Disclosure of Interest None Declared.

\section{REFERENCE}

1. Westaby, D. et al. The provision of a percutaneously placed enteral tube feeding service. Gut 2010; 59: 1592-1605. 